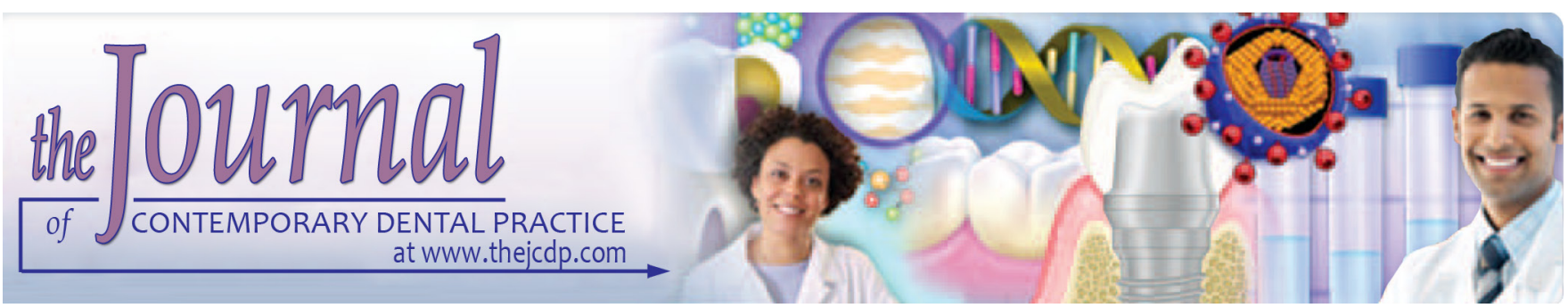

\title{
Evaluation of Treatment Outcome Following Closed Reduction of Nasal Bone Fractures
}

\author{
${ }^{1}$ T Ajay Das, ${ }^{2}$ A Sachin Aslam, ${ }^{3}$ Ummar Mangalath, ${ }^{4}$ Roshni Abida, ${ }^{5}$ Rakesh B Nair, ${ }^{6}$ Sooraj Soman
}

\begin{abstract}
Introduction: The closed reduction of a displaced nasal fracture is a preferred method in oral and maxillofacial surgery. This prospective study was conducted to evaluate the treatment outcome following closed reduction of nasal bone fractures.
\end{abstract}

Materials and methods: A total of 20 patients with nasal bone fracture who underwent closed reduction were included in the study. The cases were operated under local or general anesthesia. The outcome of treatment was evaluated pre- and postoperatively through systematic follow-ups. Clinical assessment was done to evaluate functional (airway patency, nasal obstruction, crepitus) as well as esthetic parameters (facial symmetry, swelling, and nasal deviation). Functional and esthetic satisfaction of patients was assessed using visual analog scale (VAS) pre- and post-operatively.

Results: It was observed that there was significant improvement in both functional and esthetic parameters following closed reduction of nasal fractures.

Conclusion: The present study suggested that closed reduction of nasal bone fracture is very effective in the management of nasal bone fractures. However, further studies with larger sample size in different clinical situations should be considered to confirm the efficacy of the same.

Clinical significance: Closed reduction can be a viable and more conservative alternative in management of nasal fractures.

Keywords: Closed reduction, Efficacy of closed reduction, Esthetics, Maxillofacial trauma, Nasal fracture.

How to cite this article: Das TA, Aslam AS, Mangalath U, Abida R, Nair RB, Soman S. Evaluation of Treatment Outcome Following Closed Reduction of Nasal Bone Fractures. J Contemp Dent Pract 2018;19(10):1174-1180.

\footnotetext{
${ }^{1-6}$ Department of Oral and Maxillofacial Surgery, MES Dental College, Malappuram, Kerala, India

Corresponding Author: T Ajay Das, Department of Oral and Maxillofacial Surgery, MES Dental College, Malappuram, Kerala India, e-mail: ajaydast@gmail.com
}

\section{Source of support: Nil \\ Conflict of interest: None}

\section{INTRODUCTION}

The most common facial fracture and third most common fracture of the human skeleton is nasal fracture comprising of $39 \%$ of the patients with maxillofacial trauma, ${ }^{1}$ caused by motor vehicle accidents, sports injuries, and physical altercations. Most of the injuries in the pediatric and geriatric group, both in males and females, result from falls and accidents. ${ }^{2}$ Nasal fractures usually are not promptly diagnosed, as they occur along with multiple facial trauma. This results in inadequate therapy of the nasal pyramid, thereby affecting esthetics and functions due to secondary nasal deformities and chronic nasal obstructions respectively. There is no consensus regarding use of closed vs open techniques, timing of repair and use of local vs general anesthesia for management of fractures of nasal complex. In view of high incidence of nasal fractures, demands for cost-effectiveness, decreased treatment time, and less bed occupancy, closed reduction under local anesthesia is becoming a lucrative option. However, use of a closed or open approach usually depends on the extent of the injury.

Some of the advantages of closed reduction are that the technique is simple, safe, easy to perform, and minimal potential morbidity. ${ }^{3}$ The closed reduction attempts to approximate and align the cartilaginous and skeletal nasal structures to their pretrauma state and to improve the airway. ${ }^{4}$ This is the technique of choice for acute isolated nasal fractures with minimum injury to bone and septum and the surgery is to be carried out in about 10 days after injury.

A fact that needs to be considered is that the nasal septum determines the position of nasal bones. Hence, patients who have an acute and isolated nasal injury 
without the involvement of septum can best be treated by closed reduction under local anesthesia in the outpatient clinic. But when the septum is involved, the patient needs to be operated under general anesthesia in the operating room under general anesthesia to correct the septum. Also, a proper follow-up determines the prognosis of nasal bone injuries. 5,6

Nose plays an important role in facial esthetics. This fact needs to be considered in nasal fractures. Review of photographs prior to nasal injury will be helpful for a proper understanding of how the patient's nose appeared before the injury. ${ }^{7}$ A gentle palpation in the nasal area is necessary to detect areas of tenderness and step offs. A tender, palpable or visible deformity is the most significant sign of fracture. Digital manipulation of the nose can elicit if any bony crepitus is present. ${ }^{8}$ Considering all these factors, this study was conducted to assess the efficacy of nasal bone fractures managed by closed reduction by evaluating both the functional and esthetic components.

\section{MATERIALS AND METHODS}

The present study was conducted in the Department of Oral and Maxillofacial Surgery, MES Dental College and Hospital, Perinthalmanna, Kerala, India. The study was approved by Institutional Ethical Committee. Treatment procedure and possible complications were explained to the patients and written informed consent to undergo surgical correction by closed reduction and periodic follow-up was obtained prior to surgery.

Twenty patients (14 male patients and 6 females) with nasal fracture and complaint of tenderness over nose were included in the study. All patients underwent closed reduction of nasal bone fracture between 2 and 12 days after trauma. Age of the patients ranged from 19 to 56 years, with the mean age being 33.7 years. Seven patients were operated under general anesthesia and 13 patients under local anesthesia. Pediatric patients and patients with associated head injury or uncontrolled systemic illness were excluded from this study.

\section{Patient Assessment}

Evaluation of patients involved a detailed case history documentation followed by clinical examination and radiologic investigation. The case history included demographic data, patient age, chief complaint, history of injury, history of previous nasal injury (if any) and presence of nasal obstruction and nasal deformity. Meticulous recording of the history of injury was recorded, as this plays an important role in the management of nasal injury. ${ }^{5}$ The key factors evaluated in the history included the time elapsed since injury to differentiate between acute and chronic conditions, the mechanism of injury,

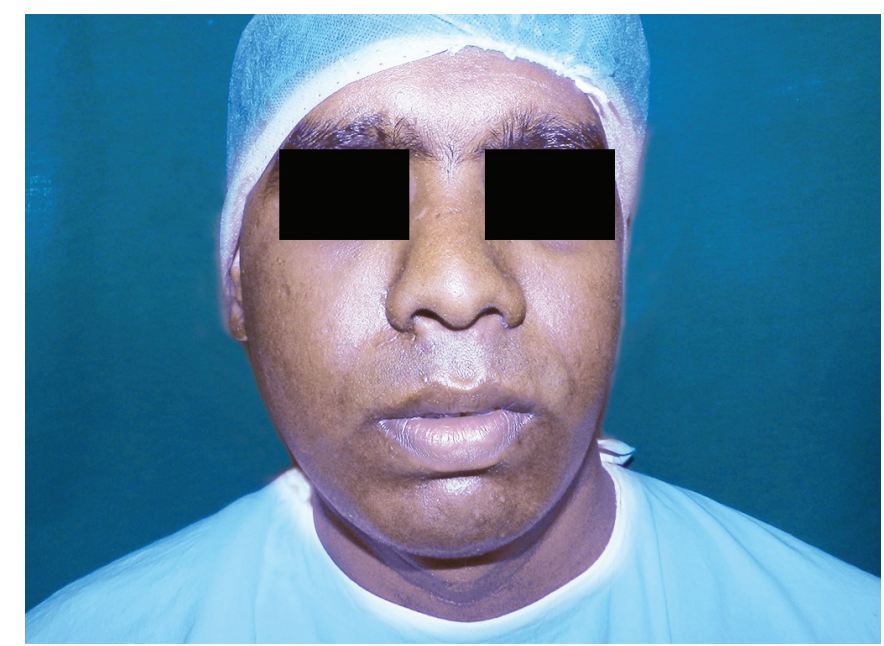

Fig. 1: Preoperative facial profile

and preexisting deformity, if any, were evaluated. Clinical examination included inspection of nose for depression or deviation, inspection and palpation of septum for the presence of deformity or deviation, checking for crepitus and tenderness in each patient (Fig. 1). Radiological examination for determination of nasal bone fracture and septal deviation included right and left true lateral nasal view (Fig. 2A), paranasal sinus view (water's view) (Fig. 2B), and computed tomography $(\mathrm{CT})$ axial view of facial bone (wherever required) (Fig. 2C).

\section{Surgical Procedure for Closed Reduction}

Closed reduction was done using digital manipulation. Seven patients were operated under general anesthesia and 13 patients under local anesthesia. Walsham's nasal reduction forceps and Asch's septal forceps were used to manipulate the nasal bone and frontal processes of maxillae and septum respectively. Local anesthesia (2\% lignocaine hydrochloride) containing 1:100,000 epinephrine was administered in order to achieve adequate anesthesia.

Manipulation of nasal bone: The small blade of the forceps was inserted into the nose and larger blade (covered by soft tubing to protect skin) was applied externally to grip the side of the nose up to medial canthus and parallel to frontal process of maxillary bone. Rotation was done medially or laterally to affect either in- or outfracture of the nasal bone with the fracture. The base of the nose was gripped between the forefinger and thumb of the opposite hand to provide additional stability prior to the actual manipulative procedure (Fig. 3A).

Manipulation of septum: The blades were introduced initially into the floor of the nose on either side of the septum and gentle pressure was used to realign the septal cartilages. The blades were gradually rotated upward and forward to grip the septum along the bridge line. Forward traction was applied to elevate the nasal bridge (Fig. 3B). 

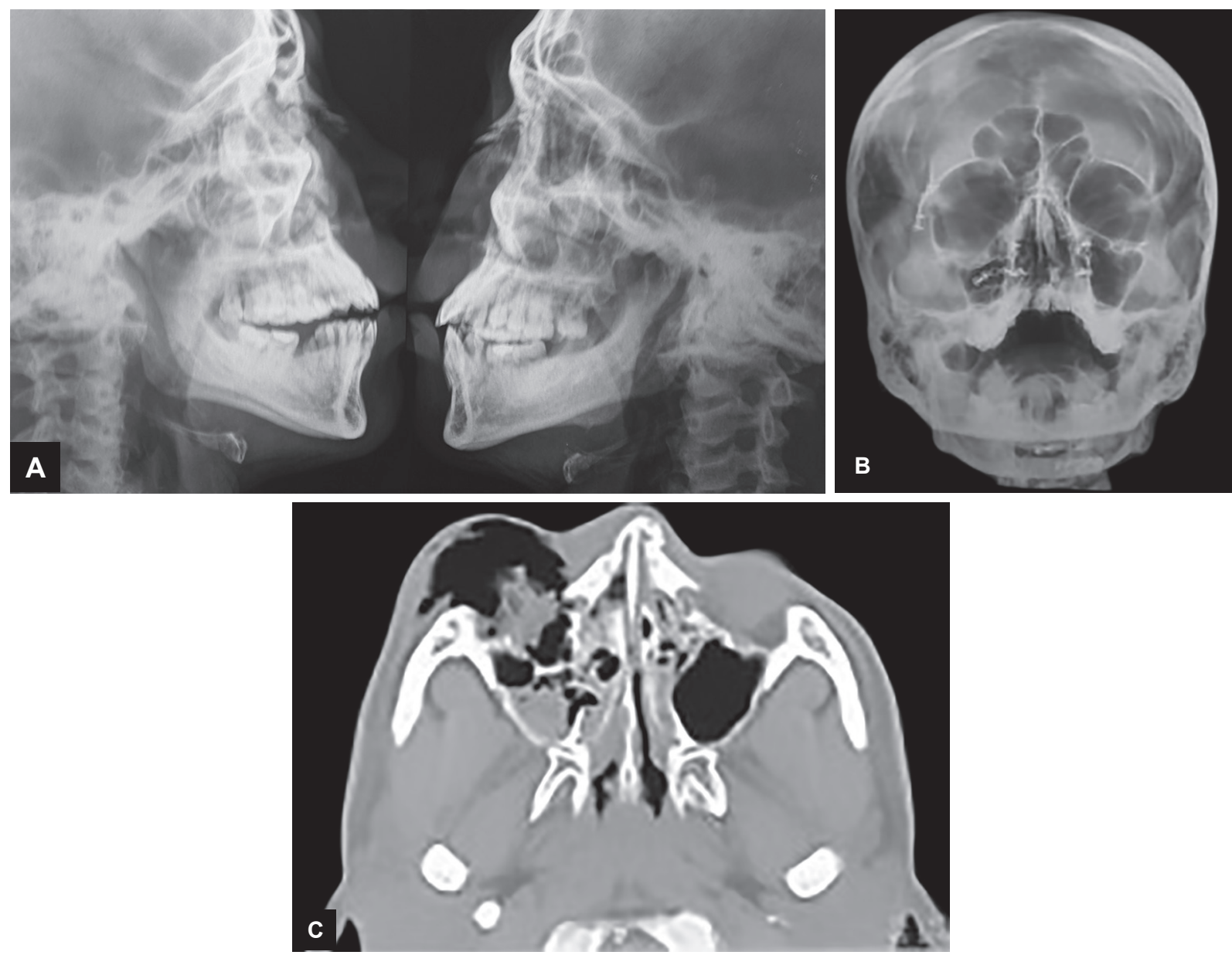

Figs 2A to C: Preoperative radiographs. (A) True nasal view; (B) Water's view; (C) CT image
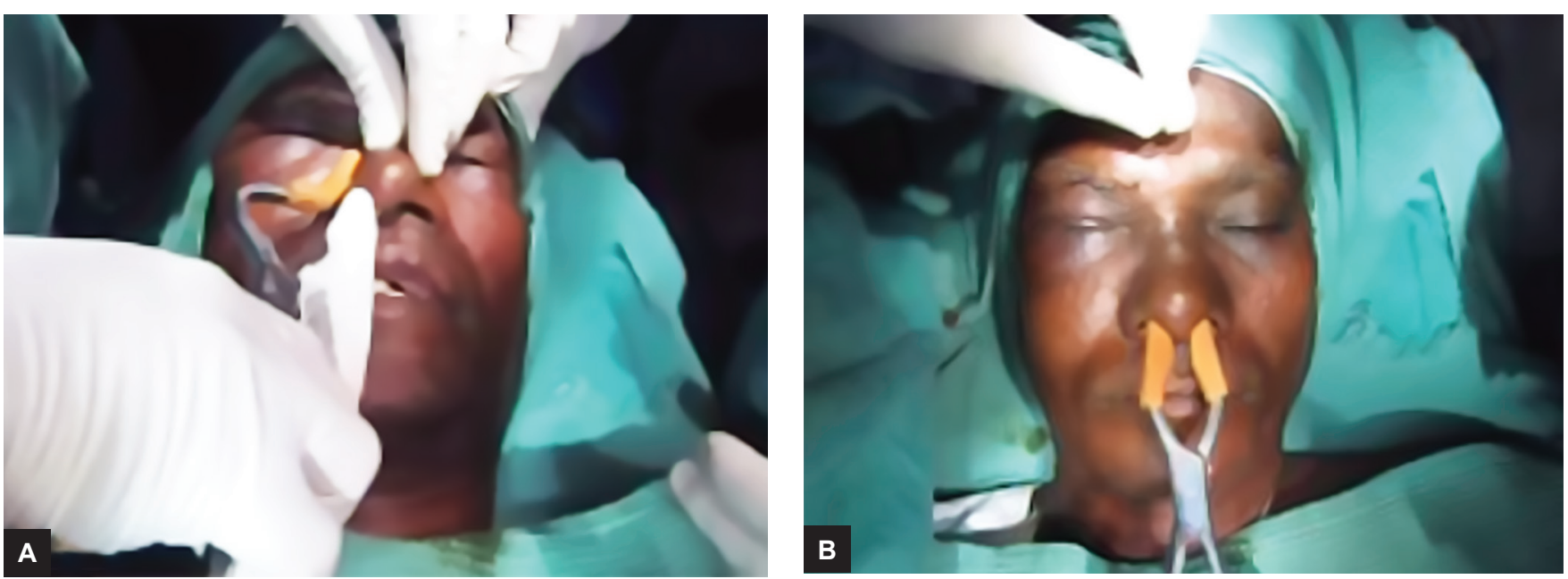

Figs 3A and B: (A) Walsham's forceps for reducing nasal bone fracture; (B) Asch's forceps for reducing septal fracture

External splinting was done using plaster of Paris splint of optimum size. Following closed reduction using forceps, the plaster was then carefully molded to the forehead, glabella, the nasal bridge line, and upper twothirds of the nose. It was ensured that plaster engaged the skin just medial to the inner canthus of each eye where it was griped between forefinger and thumb (Fig. 4A).
While plaster was setting the edges, particularly near the eyes, the area along the side and over tip of the nose was smoothened to prevent subsequent soreness of the adjacent skin. When plaster had set, it was secured to the forehead and cheeks with adhesive tapes (Fig. 4B). Patients were called on the 3rd postoperative day and splint was rechecked to rule out ill-fitting of splint. Airway 

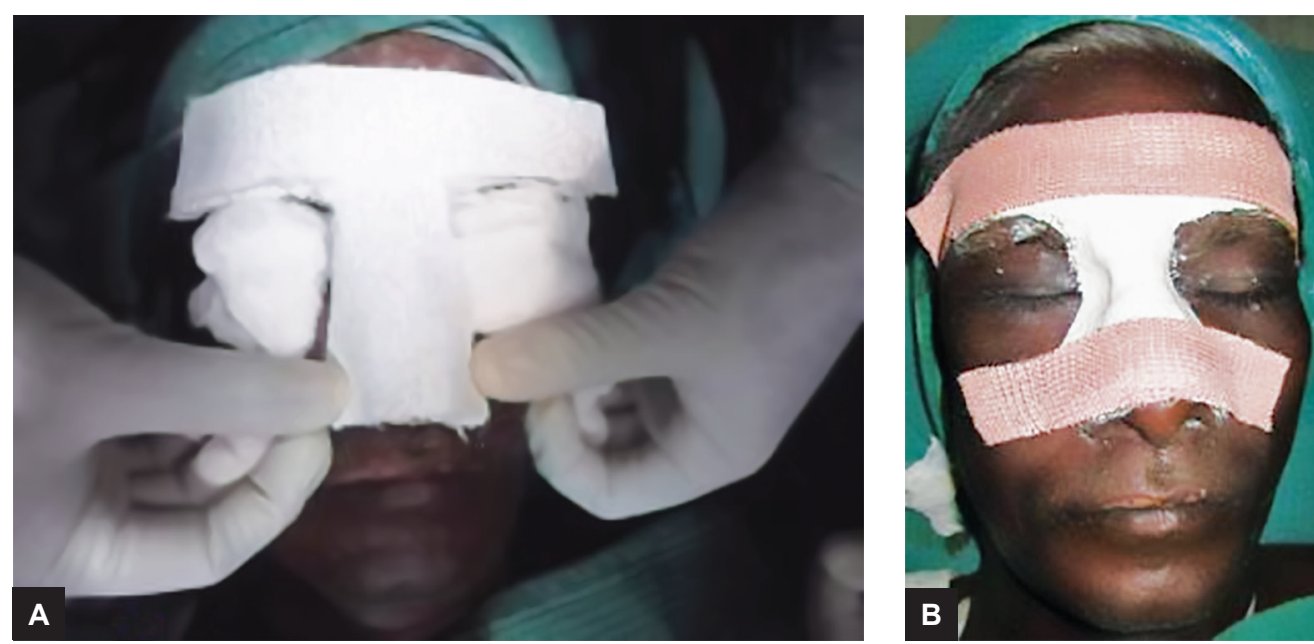

Figs 4A and B: (A) Adaptation of POP nasal stent; (B) Securing it with compression plaster

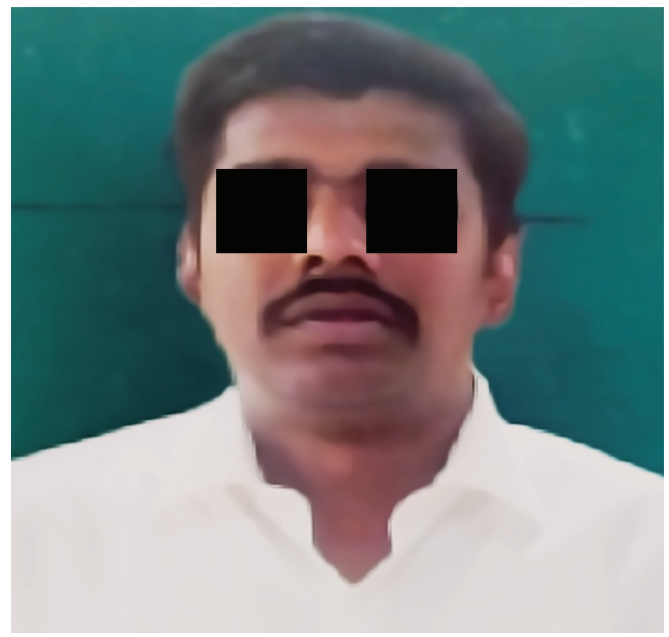

Fig. 5: Postoperative facial profile

patency and nasal obstruction were assessed pre- and postoperatively to evaluate the functional outcome of the treatment.

\section{POSTOPERATIVE CARE}

Postoperatively patients were shifted to recovery room and their vitals were monitored. Antibiotics and analgesic were advised per oral for 7 days. Patients were reviewed postoperatively both clinically and with help of radiographs to evaluate the level of bone healing. External nasal splint was removed on the 14th day of surgery. Nose was examined by the surgeon for any secondary nasal deformity or any other complication. Preoperative and postoperative photographs (Fig. 5) and radiographs were compared to evaluate the outcome and bone healing following the procedure. Patients were asked to reassess their present nasal shape after surgery and compare it with pretrauma and before surgery. Following treatment, patients were asked to evaluate the esthetic and functional components and rate the outcome of treatment on a VAS having scores from 1 to 10 where 10 meant happy with outcome and 1 means unsatisfied with outcome.

\section{RESULTS}

During the execution of the procedure in the 20 patients, it was found that $30 \%(6 / 20)$ had developed bleeding intraoperatively which was managed by anterior nasal packing. Similarly, 10\% (2/20) developed signs of syncope, but did not result in loss of consciousness. Both the patients recovered within a short period of time. They required postural repositioning which was done by elevating the lower limbs to the plane of the trunk. Both these complications did not interfere in the outcome of treatment.

The highest percentage of patients were in their 2nd and 3rd decade of life (45\%), while patients above 50 years were comparatively lowest in number $(10 \%)$. Among the 20 patients examined, 14 were males $(70 \%)$ and 6 females $(30 \%)$, which showed that the incidence of nasal bone fractures were twice more common in males than in females. While assessing the cause of injury, road traffic accident was the most common (35\%), followed by assault and sports injury ( $20 \%$ each). There was no significant relationship between the effectiveness of close reduction treatment and age, gender, cause of trauma, and type of nasal fracture. Based on the type of fracture, 9 out of 20 cases had simple unilateral nasal fractures while the remaining 11 had bilateral fractures (Table 1). No case included in this study was associated with septal injury.

Data recorded were analyzed and performed with Statistical Package for the Social Sciences, version 21. Paired t-test was used to compare pre- and postoperative evaluation of functional components and also the degree of esthetic and functional satisfaction (Table 2). A p-value less than 0.05 was considered to be significant. 
Table 1: Distribution of frequencies for physical examination findings

\begin{tabular}{lll}
\hline Variable & Number & Percentage \\
\hline Type of fracture & 9 & 45 \\
Unilateral & 11 & 55 \\
Bilateral & 0 & 0 \\
Septal fracture included & & \\
Fracture displacement & 4 & 20 \\
Undisplaced & 16 & 80 \\
Displaced & 10 & \\
Deviation of nose & 6 & 50 \\
Right & & 30 \\
Left & 18 & 90 \\
Crepitus & 2 & 10 \\
Present & & \\
Absent & & \\
\hline
\end{tabular}

Table 2: Comparison of pre- and postoperative evaluation of functional components

\begin{tabular}{llll}
\hline Variable & Preoperative & Postoperative & Paired t-test \\
\hline $\begin{array}{l}\text { Airway patency } \\
\text { Present }\end{array}$ & 5 & 19 & $\mathrm{p}=0.001$ \\
Absent & 15 & 1 & $\begin{array}{l}\text { Statistically } \\
\text { significant } \\
(p<0.05)\end{array}$ \\
& & & $p=0.023$ \\
Nasal obstruction & 14 & 1 & $\begin{array}{l}\text { Statistically } \\
\text { significant } \\
(p<0.05)\end{array}$ \\
Absent & 6 & 19 &
\end{tabular}

Table 3: Degree of functional and esthetic satisfaction after closed reduction procedure

\begin{tabular}{llll}
\hline Variable & Number & Percentage & Paired t-test \\
\hline Functional satisfaction & & & \\
Happy (9-10) & 12 & 60 & $\mathrm{p}=0.001$ \\
Satisfied (5-8) & 7 & 35 & $\mathrm{p}=0.025$ \\
Unsatisfied (<5) & 1 & 5 & $\mathrm{p}=0.003$ \\
Esthetic satisfaction & & & \\
Happy (9-10) & 12 & 60 & $\mathrm{p}=0.001$ \\
Satisfied $(5-8)$ & 7 & 35 & $\mathrm{p}=0.023$ \\
Unsatisfied $(<5)$ & 1 & 5 & $\mathrm{p}=0.001$ \\
\hline
\end{tabular}

Comparison of pre- and postoperative functional components was found to be statistically significant $(\mathrm{p}<0.05)$. Following the treatment, considering both functional and esthetic components, 12 patients $(60 \%)$ were happy with the treatment and gave a score between 9 and 10 on VAS while 7 patients (35\%) were satisfied (5-8 in VAS) and one patient was not satisfied and was given appointment for open reduction later (Table 3).

\section{DISCUSSION}

The nose is the most prominent feature of the face which has increased risk of trauma even with minimal force. ${ }^{1}$
However, its protruded position in the mid-face makes it more vulnerable for blunt injury. Mostly even a minor impact in this region results in the fracture of nasal bones. This also contributes to the increased incidence of nasal bone fractures in maxillofacial trauma. Nasal fracture may result in functional or cosmetic defect even though it is considered to be a minor injury. Since earlier days, closed reduction of the fractured nose has been the standard treatment. The nasal region is highly vascular which predisposes individuals with nasal trauma to epistaxis ${ }^{4}$ and also in bleeding during surgical procedures.

In this study, nasal fractures of male patients were twice as female patients which is in concordance with studies of Faryabi et al. ${ }^{2}$ and Murray et al. ${ }^{3}$ The data from the current study are in concordance with those of Bakardjiev and Pechalova ${ }^{4}$ and Wulkan et al., ${ }^{5}$ which emphasize the predominance of male subjects having nasal fracture or injury. Also, nasal injury was predominantly seen in young males, the most common cause being road traffic accidents followed by physical assault, and trauma in sports, especially football. ${ }^{1}$ In the present study, the highest rate of nasal fracture was observed among the age group of $<30$ years $(45 \%)$ and the lowest prevalence was observed in age groups and 50 to 60 years $(10 \%)$ that is comparable with the study of Faryabi et $\mathrm{al}^{2}$ and Hwang et $\mathrm{al}^{6}$ where highest incidence of nasal fractures was among the patients in the second and third decades of life.

The closed reduction technique for the management of a displaced nasal bone fracture has been a successful treatment option for restoring the form and function of nose in the fields of ear, nose, and throat; oral and maxillofacial surgery; and plastic surgery.

It is difficult to confirm the nasal bone fracture without systematic clinical examination using radiographs alone. ${ }^{9}$ Therefore, lateral view of nasal bone and waters view were taken as a part of routine examination. ${ }^{10}$ To arrive at a confirmatory diagnosis, also CT and its three-dimensional reformatting ${ }^{11}$ aid in more accurate and precise diagnosis. ${ }^{12}$ In the present study, CT scan was taken in $40 \%$ of cases to assess multiple facial bone fractures.

The degree and severity of injury dictate the management of nasal trauma. The ideal candidates for closed reduction technique are patients with isolated nasal bone fractures (unilateral or bilateral) and minimally deviated nasal septal complex fracture. Murray et al. ${ }^{3}$ have suggested that in the cases of nose being deviated acutely by more than half of its width from the midline, septum would be likely involved in trauma. In all the cases in this study, the nasal septum deviation was less than half of its width from midline, which was suggestive that there was no involvement of nasal septum. Within the first few 
hours of the injury, it is easy for the surgeon to perform closed reduction as the swelling will be minimal, which allows proper visibility of the defect. Once the swelling develops or the patient reports late, intervention is mostly deferred till the swelling subsides. This time period varies with the extent of the nasal injury and the impact. Closed reduction should be done generally between 2 and 12 days following the trauma; 5.3 days was the average time taken for closed reduction in this study. ${ }^{13}$

In this study, 13 cases were done under local anesthesia (LA) and seven cases under general anesthesia (GA). Many authors have suggested that closed reduction under LA to be as effective as under GA and considered it as the first line of management of nasal fractures. The simplest approach for the closed reduction of fractured nasal bones to their normal position (preinjury state) is accomplished by refracturing the bony segments and digitally manipulating under LA. If the fractured nasal bones are impacted or unable to reduce by this method, then we have to consider the use of specialized instruments designed for his purpose called Walsham's forceps and Asch's forceps. Walsham's forceps are used for reducing the nasal bone fractures, while Asch's forceps are primarily used in reducing the deviated nasal septum. However, both these instruments should be used with caution, as its inadvertent use can result in irreversible damage to the nasal mucosa.

It is also safe, efficient, and also avoids the risks associated with GA. ${ }^{14-16}$ This correlates with our study in which there was no difference in clinical outcomes for cases done under LA with cases done under GA. Manipulation under LA was also quicker to perform and did not require hospitalization resulting in freeing up of resources and theatre time. ${ }^{17,18}$

The fractured nasal bones have a tendency to move after repositioning due to minor trauma or drift back to their pre-reduction position. Hence, it is important to review the patients and keep them on regular follow-up following nasal fracture reduction. ${ }^{19,20}$ Patients in this study were followed up on a regular basis for a duration of 6 months.

\section{CONCLUSION}

Closed reduction of nasal bone fracture in the present study including 20 patients when evaluated both clinically and using radiographs suggests that this technique is very effective and produces excellent results in the cases with nasal bone fractures. Closed reduction is an easy and sufficient treatment for nasal fractures, especially for mild nasal fractures and its early intervention raises the patient satisfaction rate. But this method is relatively technique-sensitive, and therefore, it is recommended that every patient suffering from nasal bone fractures must be evaluated individually by thorough clinical and radiographic examination and by taking appropriate $\mathrm{CT}$ scan and/or radiographs, if needed, before attempting closed reduction techniques.

However, further studies with larger sample size in different clinical situations should be considered to authenticate the efficacy of the same.

\section{REFERENCES}

1. Vilela F, Granjeiro R, Junior CM, Andrade P. Applicability and effectiveness of closed reduction of nasal fractures under Local Anesthesia. Int Arch Otorhinolaryngol 2014 Jul;18(3): 266-271.

2. Faryabi J, Enhesari A, Sigari P, Pouradeli S. Efficacy of closed reduction surgery on the treatment of nasal bone fracture in admitted patients to Shahid Bahanor Hospital, Kerman, Iran. J Oral Health Oral Epidemiol 2016 Sep;5(3):148-153.

3. Murray JA, Maran AG, Busuttil A, Vaughan G. A pathological classification of nasal fractures. Injury 1986 Sep;17(5):338-344.

4. Bakardjiev A, Pechalova P. Maxillofacial fractures in Southern Bulgaria - a retrospective study of 1706 cases. J Craniomaxillofac Surg 2007 Apr;35(3):147-150.

5. Wulkan M, Parreira JG Jr, Botter DA. Epidemiologia do trauma facial. Rev Assoc Med Bras 2005;51(5):290-295.

6. Hwang K, You SH, Kim SG, Lee SI. Analysis of nasal bone fractures: a six-year study of 503 patients. J Craniofac Surg 2006 Mar;17(2):261-264.

7. Fattahi T, Steinberg B, Fernandes R, Mohan M, Reitter E. Repair of nasal complex fractures and the need for secondary septorhinoplasty. J Oral Maxillofac Surg 2006 Dec;64(12):1785-1789.

8. Moore PM Jr, Harris HE. Fractures of the nasal bones. Am J Surg 1940 Dec;50(3):668-671.

9. Chan J, Most SP. Diagnosis and management of nasal fractures. Oper Tech Otolaryngol 2008 Dec;19(4):263-266.

10. Gharehdaghi J, Rad BS, Samani VG, Kolahi F, Zonoozian AK, Marashian SM. Comparison of physical examination and conventional radiography in diagnosis of nasal fracture. Indian J Otolaryngol Head Neck Surg 2013 Aug;65(Suppl 2):S304-S307.

11. Bartkiw TP, Pynn BR, Brown DH. Diagnosis and management of nasal fractures. Int J Trauma Nurs 1995 Jan-Mar;1(1):11-18.

12. Atighechi S, Baradaranfar MH, Akbari SA. Reduction of nasal bone fractures: a comparative study of general, local, and topical anesthesia techniques. J Craniofac Surg 2009 Mar;20(2):382-384.

13. Kim HS, Lee HK, Jeong HS, Shin HW. Decreased postoperative pain after reduction of fractured nasal bones using a nerve block of the anterior ethmoidal nerve. Int J Oral Maxillofac Surg 2013 Jun;42(6):727-731.

14. Yilmaz MS, Guven M, Varli AF. Nasal fractures: is closed reduction satisfying? J Craniofac Surg 2013 Jan;24(1):e36-e38.

15. Mondin V, Rinaldo A, Ferlito A. Management of nasal bone fractures. Am J Otolaryngol 2005 May-Jun;26(3):181-185.

16. Zimmermann CE, Troulis MJ, Kaban LB. Pediatric facial fractures: recent advances in prevention, diagnosis and management. Int J Oral Maxillofac Surg 2005 Jan;34(1):2-13.

17. Higuera S, Lee EI, Cole P, Hollier LH Jr, Stal S. Nasal trauma and the deviated nose. Plast Reconstr Surg 2007 Dec;120(Suppl 2): 64S-75S. 
18. Ridder GJ, Boedeker CC, Fradis M, Schipper J. Technique and timing for closed reduction of isolated nasal fractures: A retrospective study. Ear Nose Throat J 2002 Jan;81(1):49-54.

19. Song SW, Jun BC, Chae SR, Kim BG. Clinical utility of threedimensional facial computed tomography in the treatment of nasal bone fractures: a new modality involving an air-bone view with a volume rendering technique. Indian J Otolaryngol Head Neck Surg 2013 Aug;65(Suppl 2):210-215.

20. Cook JA, McRae RD, Irving RM, Dowie LN. A randomized comparison of manipulation of the fractured nose under local and general anaesthesia. Clin Otolaryngol Allied Sci 1990 Aug;15(4):343-346. 